\section{PHYSICS REVIEW}

\author{
A.A. Konstas \\ G.V. Goldmakher \\ T.-Y. Lee \\ M.H. Lev
}

\title{
Theoretic Basis and Technical Implementations of CT Perfusion in Acute Ischemic Stroke, Part 1: Theoretic Basis
}

\begin{abstract}
SUMMARY: CT perfusion (CTP) is a functional imaging technique that provides important information about capillary-level hemodynamics of the brain parenchyma and is a natural complement to the strengths of unenhanced CT and CT angiography in the evaluation of acute stroke, vasospasm, and other neurovascular disorders. CTP is critical in determining the extent of irreversibly infarcted brain tissue (infarct "core") and the severely ischemic but potentially salvageable tissue ("penumbra"). This is achieved by generating parametric maps of cerebral blood flow, cerebral blood volume, and mean transit time.
\end{abstract}

$\mathbf{P}$ art 1 of this review establishes the clinical context of CT perfusion (CTP). Next, a discussion follows on CTP map construction by using the maximal slope method and the 2 main deconvolution techniques, Fourier transformation (FT) and singular value decomposition (the latter being the most commonly used numeric method in CTP). Part 2 discusses the pearls and pitfalls of CTP map acquisition, postprocessing, and image interpretation. Issues including radiation dose- reduction strategies, methods of correcting arterial input function (AIF) delay, the effect of laterality of AIF choice, vascular pixel elimination, the importance of correct cerebral blood flow (CBF) and cerebral blood volume (CBV) threshold selection, and the selection of appropriate perfusion parameters for correct estimation of penumbra are addressed. The review highlights the need for validation and standardization of important CTP parameters to improve patient outcomes and to design future randomized clinical trials that will provide evidence for the importance of the core/penumbra "mismatch" in patient triage for recanalization therapies beyond the current 3-hour therapeutic window for intravenous thrombolysis.

\section{Clinical Context of CTP}

The management of acute ischemic stroke remains challenging because there is a limited time window in which diagnosis must be made and therapy administered. Intravenous tissue plasminogen activator (tPA), to be used within 3 hours of stroke onset based on the 1995 National Institute of Neurological Disorders and Stroke trial, and the Mechanical Embolus Removal in Cerebral Ischemia clot retrieval device, to be used within 9 hours of stroke onset, are the only treatments currently approved by the US Food and Drug Administration for acute stroke. ${ }^{1-8}$ The only imaging technique currently required before intravenous tPA administration is an unenhanced head CT, used to exclude intracranial hemorrhage (an absolute contraindication) and infarct size greater than one third of the middle cerebral artery (MCA) territory (a relative contraindication and predictor of increased hemorrhagic risk

From the Department of Radiology (A.A.K., G.V.G., M.H.L.), Massachusetts General Hospital, Boston, Mass; and Imaging Research Laboratories (T.-Y.L.), Robarts Research Institute, London, Ontario, Canada.

Please address correspondence to Angelos A. Konstas, MD, Department of Radiology, Massachusetts General Hospital, 55 Fruit St, Boston, MA 02114; e-mail: akonstas@partners.org

DOI 10.3174/ajnr.A1487 following tPA administration). ${ }^{9,10}$ The strict 3-hour therapeutic window from symptom onset, delays in transportation and triage, and the multiple contraindications to tPA administration, however, all limit the use of intravenous tPA to typically $3 \%-5 \%$ of patients admitted with ischemic stroke. ${ }^{11}$

There has been increasing interest in advanced CT and MR imaging techniques to extend the traditional anatomic applications of imaging and offer additional insights into the pathophysiology of acute stroke. A wider time-to-treatment window might be achieved in patients who demonstrate a "mismatch" in the size of the ischemic "core" of irreversibly infarcted tissue and hypoperfused "penumbra" (the severely ischemic but potentially salvageable tissue). ${ }^{12-14}$ There is increasing evidence that core/penumbra mismatch in some patients may persist up to 12 or even 24 hours from the ischemic insult. ${ }^{15,16}$ Hence, the judicious use of intravenous tPA and endovascular therapy beyond 6-9 hours, by using advanced imaging for patient selection, is currently under intense study. Acute stroke imaging addresses 4 critical questions ${ }^{17}$ :

- Is there hemorrhage?

- Is there intravascular thrombus that can be targeted for thrombolysis?

- Is there a core of critically ischemic irreversibly infarcted tissue?

- Is there a penumbra of severely ischemic but potentially salvageable tissue?

CTP addresses the last 2 questions, after unenhanced CT and CT angiography (CTA) have each addressed the first and the second questions, respectively. The application of CTP was first proposed as early as 1980 by Axel $^{18}$; however, the CT acquisition and postprocessing systems available at that time were too slow to make CTP a practical reality.

Cerebral perfusion refers to tissue (capillary)-level blood flow. Brain-tissue flow can be described by several parameters, including $\mathrm{CBF}, \mathrm{CBV}$, and mean transit time (MTT) (Fig 1). $\mathrm{CBV}$ is defined as the total volume of flowing blood in a given volume in the brain, with units of milliliters of blood per $100 \mathrm{~g}$ of brain tissue. CBF is defined as the volume of blood moving through a given volume of brain per unit time, with units of milliliters of blood per $100 \mathrm{~g}$ of brain tissue per minute. MTT is defined as the average transit time of blood through a given brain region, measured in seconds. "Core" is typically operationally defined as the CBV lesion volume, and "penumbra," 

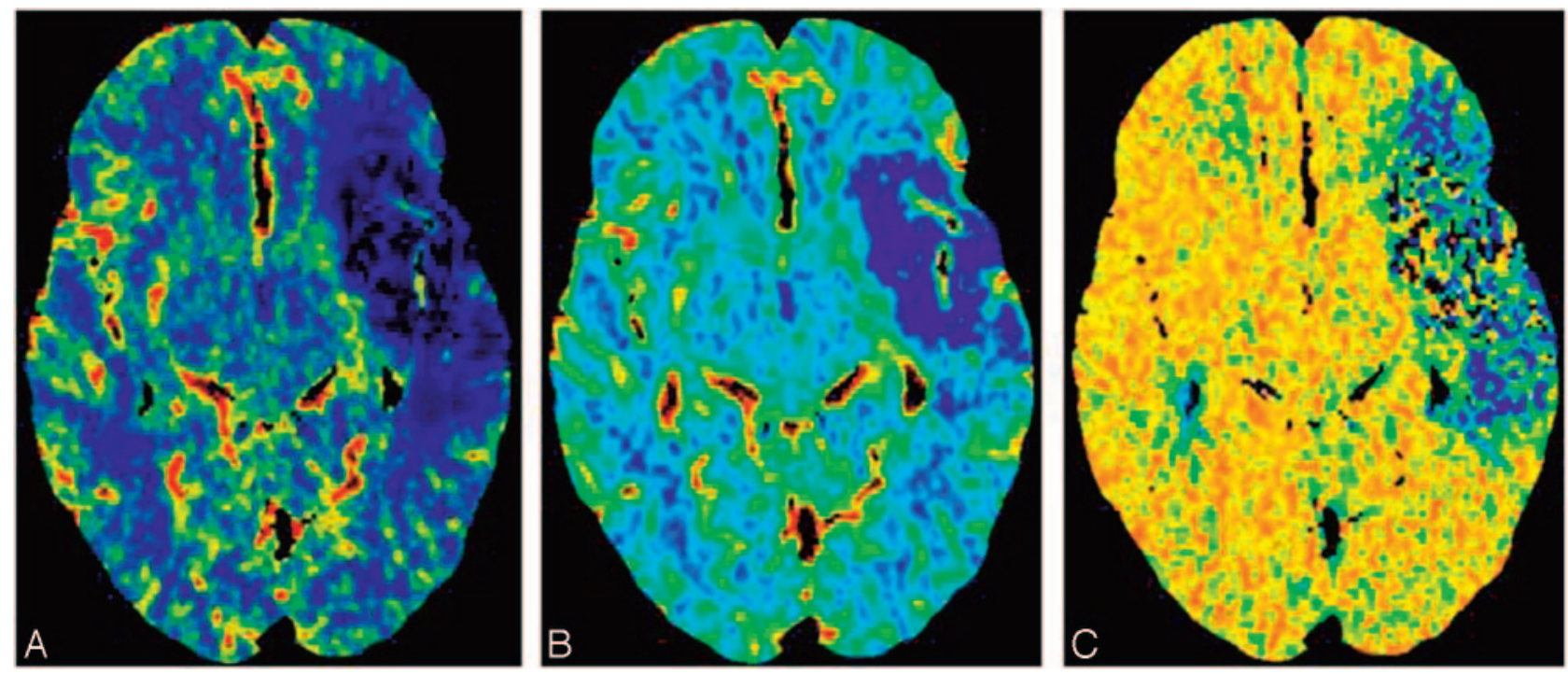

Fig 1. CT perfusion images obtained in a patient with acute ischemic stroke demonstrate a large perfusion defect in the left MCA distribution, with minimal CBV/MTT or CBF mismatch A, CBF. B, CBV. C, MTT.

as the MTT or CBF lesion volume. ${ }^{17}$ "Mismatch" is typically defined, therefore, as the difference between these.

The list of completed and ongoing trials by using core/ penumbra mismatch as a selection criterion for treatment is long and illustrates the increasingly important role of advanced imaging in acute stroke management. These trials use predefined threshold values for core and penumbra to select patients who can potentially benefit from recanalization. Completed trials include Desmoteplase in Acute Ischemic Stroke (DIAS), ${ }^{12}$ Dose Escalation of Desmoteplase for Acute Ischemic Stroke (DEDAS), ${ }^{19}$ Diffusion and Perfusion Imaging Evaluation for Understanding Stroke Evolution (DEFUSE), ${ }^{20}$ the German Multicenter Trial, ${ }^{21}$ and Echoplanar Imaging Thrombolysis Evaluation Trial (EPITHET) ${ }^{22}$; continuing trials include MR and Recanalization of Stroke Clots by using Embolectomy (MR RESCUE). ${ }^{23}$ CT perfusion can also be applied with nonrecanalization-based neuroprotective therapies for acute stroke.

In present clinical practice, CTP can be used to identify patients with MCA occlusion and core/penumbra mismatch for induced hypertension therapy with phenylephrine (Neosynephrine), even beyond the thrombolysis window. ${ }^{24}$ Highflow oxygen therapy (hyperbaric or normobaric) is another promising neuroprotective strategy for which patient selection with mismatch imaging may play a role. ${ }^{25,26}$ Patients in intensive care units who have undergone cardiac surgery might also benefit from CTP; a recent study reported greater sensitivity in detecting and mapping acute ischemic stroke in these patients in whom conventional imaging findings were inconsistent with the severity of clinical condition. ${ }^{27}$

Secondary cerebral infarction due to subarachnoid hemorrhage (SAH) - related vasospasm is the most significant cause of mortality and morbidity following aneurysm rupture. ${ }^{28}$ Transcranial Doppler sonography, CTA, and cerebral angiography can all detect angiographic spasm, the visually apparent reduction in vessel caliber. They cannot, however, detect clinical spasm (the syndrome of confusion and decreased level of consciousness resulting from decreased blood flow to the

\begin{tabular}{lc}
\hline \multicolumn{2}{l}{ Table 1: Comparison of CTP with MRP imaging } \\
\hline CTP & MRP \\
\hline $\begin{array}{lc}\text { CTP advantages } \\
\text { Linear relation of signal } \\
\text { changes with contrast }\end{array}$ & $\begin{array}{c}\text { Nonlinear relation of signal } \\
\text { changes with gadolinium }\end{array}$ \\
concentration; quantitative & concentration; nonquantitative \\
maps & maps \\
Higher spatial resolution & Lower spatial resolution \\
More readily available & Not as readily available \\
& MRP advantages \\
lonizing radiation & No ionizing radiation \\
Limited z-direction coverage & Whole-brain coverage
\end{tabular}
lodinated contrast-related concerns

Complex postprocessing

Less labor-intensive postprocessing

Note:-NSF indicates nephrogenic systemic fibrosis; CTP, CT perfusion; MRP, MR perfusion.

brain). CTP is more sensitive in detecting clinically relevant spasm, measured as a decrease in CBF and delay in MTT, ${ }^{29}$ than the methods used for angiographic spasm. ${ }^{30}$ Several studies have demonstrated that CTP is a sensitive and early predictor of secondary cerebral infarction in both patients and animal models with SAH-related clinical spasm..$^{28,30-32}$

\section{CTP Theory and Modeling}

\section{Comparison with MR Perfusion-Weighted Imaging}

CTP imaging techniques are relatively new compared with MR imaging-based methods; their clinical applications are, therefore, less thoroughly reported in the literature. ${ }^{33-35} \mathrm{De}-$ spite this, because the general principles underlying the computation of perfusion parameters such as $\mathrm{CBF}, \mathrm{CBV}$, and MTT are the same for both MR imaging and CT, the overall clinical applicability of perfusion imaging by using either of these techniques is similar. Differences in technique create several important distinctions, however, summarized in Table 1. The most important advantage of CTP is the linear relationship between contrast concentration and attenuation in CT, which 
facilitates quantitative (versus relative) measurement of CBF and CBV. MR perfusion imaging (MRP) relies on the indirect $\mathrm{T} 2{ }^{\star}$ effect induced in the tissue by gadolinium; the T2* effect itself is not linearly related to the gadolinium concentration, making absolute measurement of CBF and CBV difficult. One disadvantage of CTP is, until recently, the relatively limited coverage, whereas MRP is capable of covering the whole brain during a single bolus injection. For making stroke-related treatment decisions, CTP coverage must be sufficient to scan the entire ischemic territory at risk of infarction. ${ }^{36}$ A second disadvantage of using CT rather than MR imaging for stroke assessment is the decreased sensitivity for detection of cerebral microbleeds compared with gradient-echo sequences. Microbleeds detected on $\mathrm{T}^{\star}{ }^{\star}$-weighted MR imaging, however, have been shown not to be a contraindication to thrombolysis. ${ }^{37}$

\section{CBV Calculations}

The dynamic first-pass approach to CTP measurement involves the intravenous administration of an intravascular contrast agent, which is tracked with serial imaging during its first circulation through the brain tissue capillary bed. The main assumption of dynamic first-pass contrast-enhanced CTP models is that the perfusion tracer is not diffusible, neither metabolized nor absorbed by the tissue through which it traverses. This is certainly the case in a healthy human brain; however, breakdown of the blood-brain barrier (BBB) in infection, inflammation, or tumor adds an additional level of complexity. When extensive BBB breakdown exists, leakage of contrast material into the extravascular space results in overestimation of CT CBV. ${ }^{38}$

The determination of cerebral perfusion by using CTP is based on examining the relationships between the arterial, tissue, and the venous enhancement. More specifically, tracer kinetic theory states that if one knows the input and the output of a tracer from a voxel, one can determine the volume of distribution (ie, fractional vascular volume) and the clearance rate (ie, flow per unit tissue volume). ${ }^{18,39,40}$ The fractional vascular volume, $f$, is defined by the following equation:

$$
f=\frac{V_{\text {vasc }}}{V_{\text {vasc }}+V_{\text {interstitium }}+V_{\text {cells }}}=\frac{V_{\text {vasc }}}{V},
$$

where $V_{\text {vasc }}, V_{\text {interstitium }}$, and $V_{\text {cells }}$ are the volumes occupied by the vascular space, interstitium, and cells, respectively. If the chosen region of interest is devoid of major blood vessels, the measured change in the CT number will reflect the tissue blood pool. The contrast concentration in the tissue, $C_{\text {tissue }}$, which is measured by the CT scanner, is smaller than the intravascular concentration, $C_{\text {vasc }}$, by the fraction $f$,

$$
C_{\text {tissue }}=f \cdot C_{\text {vasc }} \text {. }
$$

The total amount of contrast material delivered to the tissue via the arteries is the product of the CBF times the integral of the arterial concentration, $C_{\text {artery }}(t)$. According to the conservation-of-mass principle, this total amount must be equal to the amount leaving the tissue, that is, the product of CBF with the integral of $C_{\text {vasc }}(t) .{ }^{18}$ Hence,

$$
C B F \cdot \int_{0}^{T} C_{\text {artery }}(t) d t=C B F \cdot \int_{0}^{T} C_{\text {vasc }}(t) d t .
$$

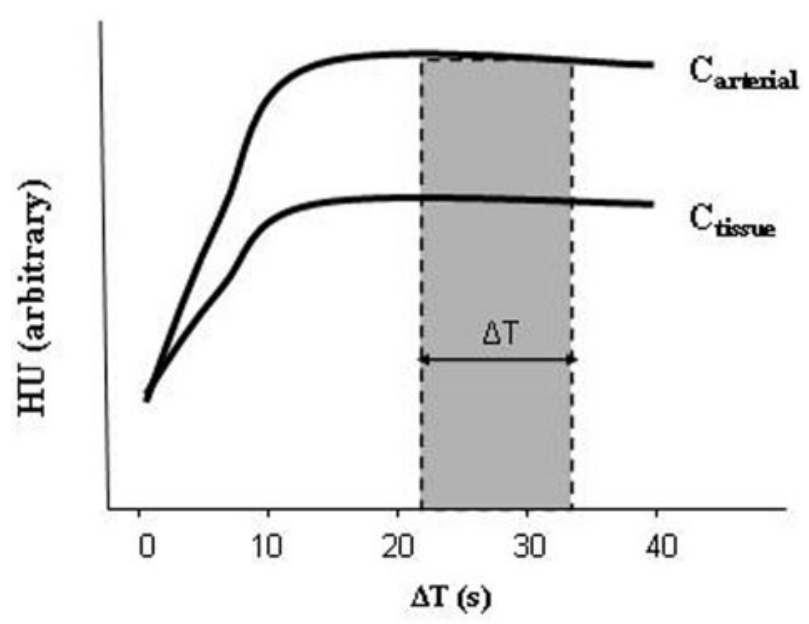

Fig 2. CTA source images acquired during a steady state of contrast concentration for both the arterial and tissue-time-attenuation curves $(\Delta \mathrm{T})$ are predominantly blood-volumerather than blood-flow-weighted. The change in attenuation due to iodine administration is directly proportional to its concentration. CBV equals the ratio of the areas under the 2 curves, $C_{\text {tissue }}$ and $C_{\text {arterial, }}$ respectively. This can be approximated as the ratio of the $\mathrm{HU}_{\text {tissue }} / \mathrm{HU}_{\text {arterial }}$ when the 2 curves approach steady state.

From equations 2 and 3, it follows that

$$
f=\frac{\int_{0}^{T} C_{\text {tissue }}(t) d t}{\int_{0}^{T} C_{\text {artery }}(t) d t} .
$$

CBV can be calculated from equation 4 if one takes into account the brain tissue attenuation, $\rho$, and a correction factor, $\mathrm{CH}$, to adjust the difference between arterial and capillary hematocrit. ${ }^{35,41}$ In vivo experiments in the 1970 s demonstrated the markedly lower hematocrit in capillaries compared with arterial hematocrit ${ }^{42-44}$; hence, the introduction of the $\mathrm{CH}$ is required for the quantification of $\mathrm{CBV}$ as follows:

$$
C B V=\frac{C H}{\rho} \cdot \frac{\int_{0}^{T} C_{\text {tissue }}(t) d t}{\int_{0}^{T} C_{\text {artery }}(t) d t} .
$$

Theoretic modeling has suggested that the source images from a CTA acquisition (CTA-SI) are predominantly bloodvolume - rather than blood-flow-weighted, assuming that a steady state of arterial and tissue contrast has been achieved during scan acquisition. ${ }^{18,35,45}$ Coregistration and subtraction of the unenhanced head CT images from the CTA-SI should, therefore, result in quantitative CBV-weighted maps. This is appealing in clinical practice because CTA-SI subtraction maps, unlike first-pass CTP maps, can provide whole-brain coverage. The change in attenuation due to iodine administration is directly proportional to its concentration; thus, the ratio of the change in Hounsfield units (HU) in brain tissue $\left(\Delta H U_{\text {tissue }}\right)$ and arterial blood $\left(\Delta H U_{\text {artery }}\right)$ can be used in practice to estimate CBV according to the relationship of equation 5 (Fig 2). Specifically, CBV can be expressed in milliliters per 
Fick Principle: Conservation of volume

- One pipe goes into the box (=artery)

- One pipe comes out of the box (=vein)

- Black box = tissue

$Q(T)=C B F \cdot \int_{0}^{T}\left[C_{\text {artery }}(t)-C_{\text {vein }}(t)\right] d t$

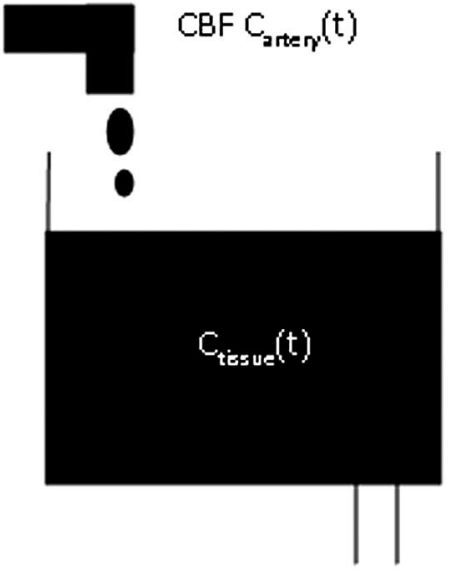

Note the assumptions!

- Singe inflow

- single outflow

- No delays (instantaneous bolus)

- No dispersion (one path)

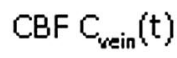

Fig 3. Fick Principle.

$100 \mathrm{~g}$ of tissue as follows:

6)

$$
C B V=\frac{\Delta H U_{\text {tissue }}}{\Delta H U_{\text {artery }}} \cdot V_{\text {voxel }} \cdot N,
$$

where $V_{\text {voxel }}$ is voxel volume and $N$ is the calculated number of voxels in $100 \mathrm{~g}$ of tissue. ${ }^{45}$

Although a relative steady state of tissue arterial contrast could be assumed with the multidetector row CT (MDCT) injection protocols used in the 1990s and early 2000s, in which low contrast-injection rates were applied with relatively long prep delay times (typically $3 \mathrm{~mL} / \mathrm{s}$, and $>25$ seconds), ${ }^{45}$ this steady-state assumption no longer holds in this era of newer faster MDCT CTA protocols, such as those used in 64-section scanners, in which injection rates $\leq 7 \mathrm{~mL} / \mathrm{s}$ and short prep delay times of 15-20 seconds change the temporal shape of the time-attenuation infusion curve, eliminating a near steady state during the timing of the CTA-SI acquisition. Hence, with the current implementation of CTA protocols on faster stateof-the-art MDCT scanners, the CTA-SI maps have become more flow-weighted than volume-weighted.

\section{Mathematic Models for CBF and MTT Calculation}

The 2 major mathematic approaches involved in calculating $\mathrm{CBF}$ and MTT are the deconvolution- and nondeconvolution-based methods. Deconvolution techniques are technically more demanding and involve more complicated and time-consuming processing, whereas nondeconvolution techniques are more straightforward but depend on simplified assumptions regarding the underlying vascular architecture. As a result, the interpretation of studies based on nondeconvolution methods may be less reliable in some situations, though this has not been fully clinically validated.

\section{Nondeconvolution-Based Models}

Like deconvolution methods, nondeconvolution methods are based on the application of the Fick principle of conservation of mass to a given region of interest within the brain parenchyma (Fig 3). The accumulated mass of contrast, $Q(T)$, in a voxel of brain tissue during a time period corresponding to the complete wash in and wash out of contrast during a bolus injection (with a saline "chaser") is equal to the product of $\mathrm{CBF}$ and the time integral of the arteriovenous difference in contrast concentration:

$$
Q(T)=C B F \cdot \int_{0}^{T}\left[C_{\text {artery }}(t)-C_{\text {vein }}(t)\right] d t .
$$

One immediate simplification that would make solving this equation less computationally intensive is to assume that during the above time period, the venous concentration is zero (ie, no contrast has yet reached the venous side of the circulation, the "no venous outflow" assumption). This assumption is valid only when $T$ is $<4-6$ seconds, the minimum transit time of blood through the brain. Under this assumption, equation 7 can be simplified as follows:

$$
Q(T)=C B F \cdot \int_{0}^{T} C_{\text {artery }}(t) d t .
$$

This is known as the Mullani-Gould formulation or singlecompartment formulation. ${ }^{46}$

When rewriting equation 8 into its derivative form for easier calculation of CBF,

9)

$$
\left[\frac{d Q(t)}{d t}\right]_{t=T}=C B F \cdot C_{\text {artery }}(T),
$$

it follows that the rate of contrast accumulation will peak when the arterial concentration is maximal:

$$
\left[\frac{d Q(t)}{d t}\right]_{M a x}=C B F \cdot\left[C_{\text {artery }}(t)\right]_{M a x} .
$$

Hence, $\mathrm{CBF}$ is the ratio of the maximum slope of $Q(t)$ to the maximum arterial concentration. This is analogous to a differentiation with respect to time of the Mullani-Gould formulation and is called the "maximum slope method". The principal advantage of the maximum slope method is the simplicity and hence speed of calculation of the perfusion val- 
ues. A very high rate of contrast agent injection, however, is required-typically at least $10 \mathrm{~mL} / \mathrm{s}$ - to satisfy the no-venous-outflow assumption. ${ }^{47,48}$ These rates cannot be routinely achieved in clinical practice. The no-venous-outflow assumption is clearly an oversimplification, and this method yields relative, rather than absolute, perfusion measurements, making interpatient or interinstitutional comparison of results difficult.

\section{Deconvolution-Based Models}

Considering a bolus of contrast tracer material injected into a tissue voxel of interest, we can define the concentration $C_{\text {tissue }}(t)$ of tracer in the tissue in terms of 2 functions:

1) Residue function, $R(t)$ : a fraction of tracer is still present in the voxel of interest at time $t$ following an ideal instantaneous unit bolus injection. $R(t)$ is unitless and is equal to 1 at $t=0^{49}$;

2) AIF, $C_{\text {artery }}(t)$ : concentration of tracer in the feeding vessel to the voxel of interest at time $t$.

Calculation of CBF requires measurement of the temporal shape of both the arterial input and the tissue-time-attenuation curves. ${ }^{40,50}$ The true input into the tissue voxel of interest cannot be measured directly; in practice, the AIF is estimated from a major artery (eg, the MCA or the "top" of the internal carotid artery), with the assumption that this represents the exact input to the tissue of interest. Any delay or dispersion of the bolus introduced during its passage from the artery used for AIF estimation to the tissue of interest will introduce errors in quantification of $\mathrm{CBF} .^{51,52}$

The observed tissue-time-attenuation curve represents a combination of the effects of the AIF and the inherent tissue properties. Thus, to fit the model, the effects of the AIF on the tissue concentration curve must be removed by using a mathematic process known as "deconvolution" to derive $R(t)$, which is dependent only on the hemodynamic properties of the voxel under consideration. $R(t)$ demonstrates an abrupt (indeed instantaneous) rise, a plateau of duration equal to the minimum transit time through the tissue of interest and then decay toward baseline. It was shown by Meier and Zieler ${ }^{53}$ that the tissue concentration curve can be represented as the CBF multiplied by AIF convolved with $R(t)$ :

$$
\begin{aligned}
C_{\text {tissue }}(t)=\frac{\rho}{C H} \cdot C B F & \cdot[A I F(t) \otimes R(t)] \\
& =\frac{\rho}{C H} \cdot C B F \cdot \int_{\tau}^{t} A I F(\tau) R(t-\tau) d \tau,
\end{aligned}
$$

where " $\otimes$ " is the convolution operator, $\rho$ is the brain tissue attenuation, and $\mathrm{CH}$ is the correction factor for the capillary hematocrit levels. $C_{\text {tissue }}$ and AIF are measured directly from the time-attenuation curve from the cine CTP source images, and the problem then becomes the calculation of $C B F$ and $R(t)$. Several methods to "deconvolve" equation 11 and hence solve for $C B F$ and $R(t)$ have been proposed and are divided into 2 main categories. With parametric methods, a specific analytic expression for $R(t)$ is assumed. Assuming a specific shape for $R(t)$ imposes assumptions on the inherent tissue properties that cannot be known a priori with sufficient precision. ${ }^{50,54,55}$ Due to this limitation, the most commonly used methods have become the nonparametric ones, which do not assume a shape for $R(t)$.

Deconvolution of equation 11 is unstable, in the sense that it frequently yields nonphysiologic oscillations (ie, noise) in the computation of the solution for $R(t)$. The nonparametric approach can be further subdivided in 2 categories, which differ in the way in which they deal with noise resulting from the instability of deconvolution. ${ }^{56}$ In the first, the transform approach, the convolution theorem of the FT is used to deconvolve equation 11 . The theorem states that the FT of the convolution of 2 time-domain functions is equivalent to the multiplication of their respective FTs. ${ }^{50,57,58}$ With the convolution theorem, equation 11 can be rewritten as

$$
R(t)=\frac{1}{C B F} \cdot \mathfrak{I}^{-1}\left\{\frac{\mathfrak{I}\left[C_{\text {tissue }}(t)\right]}{\mathfrak{I}[A I F(t)]}\right\},
$$

where $\mathfrak{I}$ is the Fourier transform. $R(t)$ and $C B F$ can thus be determined by taking the inverse FT of the ratios of the 2 transforms of the sampled $A I F(t)$ and $C_{\text {tissue }}(t)$. However, this approach is very sensitive to noise, as demonstrated by Ostergaard et al. ${ }^{55}$

The second approach, the more commonly applied singular value decomposition (SVD), is an algebraic reformulation of the convolution integrals of equation 11 rewritten as

$$
\begin{aligned}
& \Delta t\left(\begin{array}{cccc}
A I F\left(t_{1}\right) & 0 & \cdots & 0 \\
A I F\left(t_{2}\right) & A I F\left(t_{1}\right) & \cdots & 0 \\
\cdots & \cdots & \cdots & 0 \\
A I F\left(t_{\mathrm{N}}\right) & A I F\left(t_{\mathrm{N}-1}\right) & \cdots & A I F\left(t_{1}\right)
\end{array}\right) \\
& \\
& \quad\left(\begin{array}{c}
R\left(t_{1}\right) \\
R\left(t_{2}\right) \\
\cdots \\
R\left(t_{\mathrm{N}}\right)
\end{array}\right)=\left(\begin{array}{c}
C_{\text {tissue }}\left(t_{1}\right) \\
C_{\text {tissue }}\left(t_{2}\right) \\
\cdots \\
C_{\text {tissue }}\left(t_{\mathrm{N}}\right)
\end{array}\right),
\end{aligned}
$$

where $t_{1}, t_{2}, \ldots t_{\mathrm{N}}$ are equally spaced time points and $A I F(t)$ and $C_{\text {tissue }}(t)$ are measured. Equation 13 can be rewritten in shorthand matrix-vector notation:

$$
A \cdot b=c,
$$

where $b$ and $c$ are vectors whose elements are $R\left(t_{i}\right)$ and $C_{\text {tissue }}\left(t_{i}\right)$, respectively, and $A$ is the convolution matrix in equation 13. It can be shown that the least squares solution for $b$ is given by $\left(\mathrm{A}^{\mathrm{T}} \cdot \mathrm{A}\right)^{-1} \cdot \mathrm{A}^{\mathrm{T}}$, where $\mathrm{A}^{\mathrm{T}}$ is the transpose of the convolution matrix and $\left(A^{T} \cdot A\right)^{-1}$ is the inverse of the symmetric matrix $A^{T} \cdot A \cdot{ }^{59}$ SVD decomposes $A$ into a product of matrices, such that $\left(A^{T} \cdot A\right)^{-1}$ can be found easily and the solution for $b$ can be written as a sum of terms weighted by the reciprocal of the singular values of $A .^{60}$ By truncating small singular values in the sum for the solution vector $b$, oscillations from noise in the both $\operatorname{AIF}(t)$ and $C_{\text {tissue }}(t)$ are avoided.

$S V D$ has yielded the most robust results from all the deconvolution methods used to map $\mathrm{CBF}^{55}$ and has gained widespread acceptance. The creation of accurate quantitative maps of CBF, CBV, and MTT by using deconvolution methods has been validated in a number of studies. ${ }^{61-67}$ Once CBF and CBV are known, MTT can be calculated from the ratio of CBV and $\mathrm{CBF}$, by using the central volume theorem. ${ }^{53,68}$

Potential pitfalls in the computation of CBF by using deconvolution methods include patient motion and partial volume averaging, which can cause underestimation of the 
Table 2: Animal and human studies validating CTP

\begin{tabular}{|c|c|c|c|c|}
\hline Study & Subjects & Validation Method & Results & Comments \\
\hline \multicolumn{5}{|l|}{ Animal Studies } \\
\hline Gobbel et al $(1991)^{69}$ & 25 Healthy dogs & Microspheres & CBF: $r=0.95, P<.05$ & \\
\hline Cenic et al (1999) $)^{61}$ & 13 Rabbits & Microspheres & CBF: $r=0.84, P<.001$, slope $=0.97$ & Deconvolution method \\
\hline Nabavi et al (1999) ${ }^{62}$ & $\begin{array}{l}5 \text { Healthy and } 7 \text { ischemic } \\
\text { beagles }\end{array}$ & Microspheres & $\begin{array}{l}\text { Healthy: } \mathrm{CBF}, r=0.78 \text {, slope }=0.93 \\
\quad \text { ischemic: } r=0.79 \text {, slope }=0.97\end{array}$ & Deconvolution method \\
\hline Cenic et al $(2000)^{64}$ & 9 Rabbits with tumors & Microspheres; & CBF: $r=0.85, P<.001$, slope $=0.99$ & Deconvolution method \\
\hline Nabavi et al $(2001)^{66}$ & 7 Ischemic rabbits & postmortem histology & $\begin{array}{l}\text { CBF: } r=0.95, \text { slope }=1.05 \\
\text { CBV: } r=0.80, \text { slope }=0.49 \\
\text { MTT: } r=0.85, \text { slope }=0.95\end{array}$ & Deconvolution method \\
\hline \multicolumn{5}{|l|}{ Human studies } \\
\hline Gillard et al $(2000)^{72}$ & $\begin{array}{l}2 \text { With gliomas, } 6 \text { with } \\
\text { AVMs }\end{array}$ & PET & $r^{2}=0.52$ & Maximal slope method; VPE \\
\hline $\begin{array}{l}\text { Wintermark et al } \\
(2001)^{67}\end{array}$ & $\begin{array}{l}9 \text { With cerebrovascular } \\
\text { disease }\end{array}$ & $\mathrm{Xe}-\mathrm{CT}$ & CBF: $r^{2}=0.79$, slope $=0.87$ & Deconvolution method \\
\hline Kudo et al $(2003)^{70}$ & 5 Healthy subjects & PET & CBF: $r=0.69$, slope $=1.05$ & Deconvolution method; VPE \\
\hline Sase et al $(2005)^{71}$ & 7 Healthy subjects & $\mathrm{Xe}-\mathrm{CT}$ & $\begin{array}{l}\text { CBF: } r^{2}=0.46-0.93 \text { depending on the } \\
\quad \text { brain region, } P<.05 \text {, slopes }<0.81 \\
\text { or }>1.20\end{array}$ & $\begin{array}{l}\text { Maximum slope method; VPE; } \\
\text { different territories compared }\end{array}$ \\
\hline Kanazawa et al $(2007)^{29}$ & 28 Healthy subjects & $\mathrm{Xe}-\mathrm{CT}$ & CBF: $r=0.61-0.70, P<.01$ & Several territories compared \\
\hline Bisdas $(2008)^{73}$ & 7 With strokes & PET & $\mathrm{CBF}: r=0.77, P=.00$ & Deconvolution method \\
\hline
\end{tabular}

Note:-AVM indicates arteriovenous malformation; PET, positron-emission tomography; Xe-CT, xenon-enhanced CT; VPE, vascular pixel elimination; CBF, cerebral blood flow; MTT, mean transit time.

$A I F(t)$. Image-coregistration software to correct for patient motion and careful choice of regions of interest for the AIF can minimize these pitfalls.

Commercial software suppliers use different mathematic methods. In the past, some have incorporated the maximum slope method, though new versions are frequently released and the reader is advised to check for the most up-to-date software from each vendor. Others have typically used deconvolution techniques, which, though theoretically superior to nondeconvolution methods - as has already and will again to be noted-the full clinical implication of using has yet to be established and standardized by the stroke imaging community.

\section{Validation of CTP}

CBF quantification with CTP has been preliminarily tested in animals and humans. In animals, the accepted reference standard method for quantifying CBF is the microsphere technique and, to a lesser extent, postmortem histology. CTP measurements have been validated in healthy animals, animals with experimental ischemic stroke, and animals with implanted tumors. ${ }^{61,62,64,66,69}$ All studies reported very good correlation between CTP and the reference-standard methods (Table 2). In humans, CTP has been validated with positron-emission tomography and xenon-enhanced CT in healthy subjects, patients with acute stroke, and patients with chronic cerebrovascular disease. Some of these studies are summarized in Table 2. Deconvolution-based CTP studies by Wintermark et $\mathrm{al}^{67}$ and Kudo et $\mathrm{al}^{70}$ gave slopes within $13 \%$ of unity, whereas the difference from unity was 20\%-60\% for the maximum slope technique used by Sase et al (2005)..$^{71}$ These results once again suggest a superior accuracy of deconvolution techniques compared with maximum slope-based methods.

\section{References}

1. Tissue plasminogen activator for acute ischemic stroke: The National Institute of Neurological Disorders and Stroke rt-PA Stroke Study Group. N Engl J Med 1995;333:1581-87
2. Furlan A, Higashida R, Wechsler L, et al. Intra-arterial prourokinase for acute ischemic stroke: The PROACT II study—a randomized controlled trial. Prolyse in Acute Cerebral Thromboembolism. JAMA 1999;282:2003-11

3. Marler JR, Tilley BC, Lu M, et al. Early stroke treatment associated with better outcome: the NINDS rt-PA stroke study. Neurology 2000;55:1649-55

4. del Zoppo GJ, Poeck K, Pessin MS, et al. Recombinant tissue plasminogen activator in acute thrombotic and embolic stroke. Ann Neurol 1992;32:78-86

5. Hacke W, Kaste M, Fieschi C, et al. Intravenous thrombolysis with recombinant tissue plasminogen activator for acute hemispheric stroke: The European Cooperative Acute Stroke Study (ECASS). JAMA 1995;274:1017-25

6. Gobin YP, Starkman S, Duckwiler GR, et al. MERCI 1: a phase 1 study of Mechanical Embolus Removal in Cerebral Ischemia. Stroke 2004;35:2848-54. Epub 2004 Oct 28

7. Katz JM, Gobin YP. Merci retriever in acute stroke treatment. Expert Rev Med Devices 2006;3:273-80

8. Smith WS, Sung G, Starkman S, et al. Safety and efficacy of mechanical embolectomy in acute ischemic stroke: results of the MERCI trial. Stroke 2005;36: 1432-38

9. Phan TG, Donnan GA, Koga M, et al. The ASPECTS template is weighted in favor of the striatocapsular region. Neuroimage 2006;31:477-81. Epub 2006 Feb 28

10. Phan TG, Donnan GA, Koga M, et al. Assessment of suitability of thrombolysis in middle cerebral artery infarction: a proof of concept study of a stereologically-based technique. Cerebrovasc Dis 2007;24:321-27. Epub 2007 Aug 9

11. Schumacher HC, Bateman BT, Boden-Albala B, et al. Use of thrombolysis in acute ischemic stroke: analysis of the Nationwide Inpatient Sample 1999 to 2004. Ann Emerg Med 2007;50:99-107. Epub 2007 May 3

12. Hacke W, Albers G, Al-Rawi Y, et al. The Desmoteplase in Acute Ischemic Stroke Trial (DIAS): a phase II MRI-based 9-hour window acute stroke thrombolysis trial with intravenous desmoteplase. Stroke 2005;36:66-73

13. Mehta N, Lev MH, Mullins ME, et al. Prediction of final infarct size in acute stroke using cerebral blood flow/cerebral blood volume mismatch: added value of quantitative first pass CT perfusion imaging in successfully treated versus unsuccessfully treated/untreated patients. In: Proceedings of the 41st Annual Meeting of the American Society of Neuroradiology, Washington, DC. April 28-May 2, 2003.

14. Murphy BD, Fox AJ, Lee DH, et al. Identification of penumbra and infarct in acute ischemic stroke using computed tomography perfusion-derived blood flow and blood volume measurements. Stroke 2006;37:1771-77

15. Darby DG, Barber PA, Gerraty RP, et al. Pathophysiological topography of acute ischemia by combined diffusion-weighted and perfusion MRI. Stroke 1999;30:2043-52

16. Neumann-Haefelin T, Wittsack HJ, Wenserski F, et al. Diffusion- and perfusion-weighted MRI: the DWI/PWI mismatch region in acute stroke. Stroke 1999;30:1591-97

17. Shetty SK, Lev MH. CT perfusion in acute stroke. Neuroimaging Clin N Am 2005; 15:481-501, ix

18. Axel L. Cerebral blood flow determination by rapid-sequence computed tomography. Radiology 1980;137:679-86

19. Furlan AJ, Eyding D, Albers GW, et al. Dose Escalation of Desmoteplase for 
Acute Ischemic Stroke (DEDAS): evidence of safety and efficacy 3 to 9 hours after stroke onset. Stroke 2006;37:1227-31

20. Albers GW, Thijs VN, Wechsler L, et al. Magnetic resonance imaging profiles predict clinical response to early reperfusion: the diffusion and perfusion imaging evaluation for understanding stroke evolution (DEFUSE) study. Ann Neurol 2006;60:508-17

21. Thomalla G, Schwark C, Sobesky J, et al. Outcome and symptomatic bleeding complications of intravenous thrombolysis within 6 hours in MRI-selected stroke patients: comparison of a German multicenter study with the pooled data of ATLANTIS, ECASS, and NINDS tPA trials. Stroke 2006;37:852-58

22. Davis SM, Donnan GA, Parsons MW, et al. Effects of alteplase beyond $\mathbf{3} \mathbf{~ h}$ after stroke in the Echoplanar Imaging Thrombolytic Evaluation Trial (EPITHET): a placebo-controlled randomised trial. Lancet Neurol 2008;7:299-309

23. Davis SM, Donnan GA, Butcher KS, et al. Selection of thrombolytic therapy beyond $3 \mathrm{~h}$ using magnetic resonance imaging. Curr Opin Neurol 2005; 18:47-52

24. Bogoslovsky T, Happola O, Salonen O, et al. Induced hypertension for the treatment of acute MCA occlusion beyond the thrombolysis window: case report. BMC Neurol 2006;6:46

25. Singhal AB. A review of oxygen therapy in ischemic stroke. Neurol Res 2007;29:173-83

26. Singhal AB, Benner T, Roccatagliata $\mathrm{L}$, et al. A pilot study of normobaric oxygen therapy in acute ischemic stroke. Stroke 2005;36:797-802

27. Bisdas S, Therapidis P, Kerl JM, et al. Value of cerebral perfusion computed tomography in the management of intensive care unit patients with suspected ischaemic cerebral pathology after cardiac surgery. Eur J Cardiothorac Surg 2007;32:521-26

28. Niikawa S, Yasokawa Y, Ito T. Development of early cerebral swelling in surgically treated ruptured aneurysm of acute stage, its significance, and management. J Stroke Cerebrovasc Dis 2005;14:58-66

29. Kanazawa R, Kato M, Ishikawa K, et al. Convenience of the computed tomography perfusion method for cerebral vasospasm detection after subarachnoid hemorrhage. Surg Neurol 2007;67:604-11

30. Pham M, Johnson A, Bartsch AJ, et al. CT perfusion predicts secondary cerebral infarction after aneurysmal subarachnoid hemorrhage. Neurology 2007;69:762-65

31. Nabavi DG, LeBlanc LM, Baxter B, et al. Monitoring cerebral perfusion after subarachnoid hemorrhage using CT. Neuroradiology 2001;43:7-16

32. Laslo AM, Eastwood JD, Pakkiri P, et al. CT perfusion-derived mean transit time predicts early mortality and delayed vasospasm after experimental subarachnoid hemorrhage. AJNR Am J Neuroradiol 2008;29:79-85

33. Roberts HC, Roberts TP, Dillon WP. CT perfusion flow assessment: "up and coming" or "off and running"? AJNR Am J Neuroradiol 2001;22:1018-19

34. Hamberg LM, Hunter GJ, Halpern EF, et al. Quantitative high resolution measurement of cerebrovascular physiology with slip-ring CT. AJNR Am J Neuroradiol 1996;17:639-50

35. Hamberg LM, Hunter GJ, Kierstead D, et al. Measurement of cerebral blood volume with subtraction three-dimensional functional CT. AJNR Am J Neuroradiol 1996;17:1861-69

36. Schaefer PW, Barak ER, Kamalin S, et al. Quantitative assessment of core/ penumbra mismatch in acute stroke: CT and MR perfusion imaging are strongly correlated when sufficient brain volume is imaged. Stroke 2008;39: 2986-92. Epub 2008 Aug 21

37. Fiehler J, Albers GW, Boulanger JM, et al. Bleeding risk analysis in stroke imaging before thrombolysis (BRASIL): pooled analysis of $2^{*}$-weighted magnetic resonance imaging data from 570 patients. Stroke 2007;38:2738-44. Epub 2007 Aug 23

38. Roberts HC, Roberts TP, Brasch RC, et al. Quantitative measurement of microvascular permeability in human brain tumors achieved using dynamic contrast-enhanced MR imaging: correlation with histologic grade. AJNR AmJ Neuroradiol 2000;21:891-99

39. Zierler KL. Equations for measuring blood flow by external monitoring of radioisotopes. Circ Res 1965;16:309-21

40. Zierler KL. Theoretical basis of indicator-dilution methods for measuring flow and volume. Circ Res 1962;10:393-407

41. Calamante F, Thomas DL, Pell GS, et al. Measuring cerebral blood flow using magnetic resonance imaging techniques. J Cereb Blood Flow Metab 1999;19: 701-35

42. Lipowsky HH, Kovalcheck S, Zweifach BW. The distribution of blood rheological parameters in the microvasculature of cat mesentery. Circ Res 1978;43:738-49

43. Klitzman B, Duling BR. Microvascular hematocrit and red cell flow in resting and contracting striated muscle. Am J Physiol 1979;237:H481-90

44. Phelps ME, Grubb RL Jr, Ter-Pogossian MM. In vivo regional cerebral blood volume by X-ray fluorescence: validation of method. J Appl Physiol 1973;35:741-47

45. Hunter GJ, Hamberg LM, Ponzo JA, et al. Assessment of cerebral perfusion and arterial anatomy in hyperacute stroke with three-dimensional functional CT: early clinical results. AJNR Am J Neuroradiol 1998;19:29-37

46. Mullani NA, Gould KL. First-pass measurements of regional blood flow with external detectors. $\mathrm{J} \mathrm{Nucl} \mathrm{Med} \mathrm{1983;24:577-81}$
47. Miles KA. Measurement of tissue perfusion by dynamic computed tomography. Br J Radiol 1991;64:409-12

48. Klotz E, Konig M. Perfusion measurements of the brain: using the dynamic CT for the quantitative assessment of cerebral ischemia in acute stroke. Eur Radiol 1999;30:170-84

49. Lee TY. Scientific basis and validation. In: Miles KA, Eastwood JD, Konig M eds. Multidetector Computed Tomography in Cerebrovascular Disease. Abingdon, UK: Informa Healthcare; 2007:13-27

50. Ostergaard L, Weisskoff RM, Chesler DA, et al. High resolution of cerebra blood flow using intravascular tracer bolus passages. Part I. Mathematical approach and statistical analysis. Magn Reson Med 1996;36:715-25

51. Calamante F, Gadian DG, Connelly A. Delay and dispersion effects in dynamic susceptibility contrast MRI: simulations using singular value decomposition. Magn Reson Med 2000;44:466-73

52. Calamante F, Gadian DG, Connelly A. Quantification of perfusion using bolus tracking magnetic resonance imaging in stroke: assumptions, limitations, and potential implications for clinical use. Stroke 2002;33:1146-51

53. Meier P, Zieler K. On the theory of the indicator-dilution method for measurement of blood flow and volume. J Appl Physiol 1954;6:731-44

54. Lassen NA. Cerebral transit of an intravascular tracer may allow measurement of regional blood volume but not regional blood flow. J Cereb Blood Flow Metab 1984;4:633-34

55. Ostergaard L, Sorensen AG, Kwong KK, et al. High resolution measurement of cerebral blood flow using intravascular tracer bolus passages. Part II. Experimental comparison and preliminary results. Magn Reson Med 1996;36: $726-36$

56. Lee T, Blake M. Implementing deconvolution analysis for perfusion CT. In Miles KA, Eastwood JD, Konig, M, eds. Multidetector Computed Tomography in Cerebrovascular Disease. Abingdon, UK: Informa Healthcare; 2007:29-45

57. Gobbel GT, Fike JR. A deconvolution method for evaluating indicator-dilution curves. Phys Med Biol 1994;39:1833-54

58. Rempp KA, Brix G, Wenz F, et al. Quantification of regional cerebral blood flow and volume with dynamic susceptibility contrast-enhanced MR imaging. Radiology 1994;193:637-41

59. Farebrother RW. Linear Least Squares Computations. New York: Marcel Dekker; 1988

60. Lawson CL, Hanson, RJ. Solving Least Squares Problems. Philadelphia: SIAM; 1995

61. Cenic A, Nabavi DG, Craen RA, et al. Dynamic CT measurement of cerebral blood flow: a validation study. AJNR Am J Neuroradiol 1999;20:63-73

62. Nabavi DG, Cenic A, Craen RA, et al. CT assessment of cerebral perfusion: experimental validation and initial clinical experience. Radiology 1999;213: 141-49

63. Wirestam R, Andersson L, Ostergaard L, et al. Assessment of regional cerebral blood flow by dynamic susceptibility contrast MRI using different deconvolution techniques. Magn Reson Med 2000;43:691-700

64. Cenic A, Nabavi DG, Craen RA, et al. A CT method to measure hemodynamics in brain tumors: validation and application of cerebral blood flow maps. AJNR Am J Neuroradiol 2000;21:462-70

65. Nabavi DG, Cenic A, Dool J, et al. Quantitative assessment of cerebral hemodynamics using CT: stability, accuracy, and precision studies in dogs. $\mathrm{J} \mathrm{Com-}$ put Assist Tomogr 1999;23:506-15

66. Nabavi DG, Cenic A, Henderson S, et al. Perfusion mapping using computed tomography allows accurate prediction of cerebral infarction in experimental brain ischemia. Stroke 2001;32:175-83

67. Wintermark M, Thiran JP, Maeder P, et al. Simultaneous measurement of regional cerebral blood flow by perfusion $C T$ and stable xenon CT: a validation study. AJNR Am J Neuroradiol 2001;22:905-14

68. Stewart GN. Researches on the circulation time in organs and on the influences which affect it. J Physiol 1894;15:1

69. Gobbel GT, Cann CE, Iwamoto HS, et al. Measurement of regional cerebral blood flow in the dog using ultrafast computed tomography: experimental validation. Stroke 1991;22:772-79

70. Kudo K, Terae S, Katoh C, et al. Quantitative cerebral blood flow measuremen with dynamic perfusion CT using the vascular-pixel elimination method: comparison with $\mathrm{H} 2(15) \mathrm{O}$ positron emission tomography. AJNR Am J Neuroradiol $2003 ; 24: 419-26$

71. Sase S, Honda M, Machida K, et al. Comparison of cerebral blood flow between perfusion computed tomography and xenon-enhanced computed tomography for normal subjects: territorial analysis. J Comput Assist Tomogr 2005;29:270-77

72. Gillard JH, Minhas PS, Hayball MP, et al. Assessment of quantitative computed tomographic cerebral perfusion imaging with $\mathrm{H} 2(15) \mathrm{O}$ positron emission tomography. Neurol Res 2000;22:457-64

73. Bisdas S, Donnerstag F, Berding G, et al. Computed tomography assessment of cerebral perfusion using a distributed parameter tracer kinetics model: validation with $\mathrm{H}(2)((15)) O$ positron emission tomography measurements and initial clinical experience in patients with acute stroke. J Cereb Blood Flow Metab 2008;28:402-11 